\title{
Predicting functionally important SNP classes based on negative selection
}

\author{
Mark A Levenstien, Robert J Klein ${ }^{*}$
}

\begin{abstract}
Background: With the advent of cost-effective genotyping technologies, genome-wide association studies allow researchers to examine hundreds of thousands of single nucleotide polymorphisms (SNPs) for association with human disease. Recently, many researchers applying this strategy have detected strong associations to disease with SNP markers that are either not in linkage disequilibrium with any nonsynonymous SNP or large distances from any annotated gene. In such cases, no well-established standard practice for effective SNP selection for follow-up studies exists. We aim to identify and prioritize groups of SNPs that are more likely to affect phenotypes in order to facilitate efficient SNP selection for follow-up studies.

Results: Based on the annotations available in the Ensembl database, we categorized SNPs in the human genome into classes related to regulatory attributes, such as epigenetic modifications and transcription factor binding sites, in addition to classes related to gene structure and cross-species conservation. Using the distribution of derived allele frequencies (DAF) within each class, we assessed the strength of natural selection for each class relative to the genome as a whole. We applied this DAF analysis to Perlegen resequenced SNPs genome-wide. Regulatory elements annotated by Ensembl such as specific histone methylation sites as well as classes defined by crossspecies conservation showed negative selection in comparison to the genome as a whole.

Conclusions: These results highlight which annotated classes are under purifying selection, have putative functional importance, and contain SNPs that are strong candidates for follow-up studies after genome-wide association. Such SNP annotation may also be useful in interpreting results of whole-genome sequencing studies.
\end{abstract}

\section{Background}

With recent technological advances, genome-wide association studies are now a reality and offer terrific promise for localizing genes responsible for complex diseases. In fact, large-scale genome-wide association studies have successfully identified genetic loci related to a wide array of disorders. Currently, over 550 publications report putative associations that have been found by this approach [1]. A benefit of this relatively unbiased strategy is the potential to identify genes in pathways previously unconnected with the disease of interest. Examples include identification of complement factor $\mathrm{H}(C F H)$ in age related macular degeneration, FGFR2 in breast cancer, and CDKN2A as well as $C D K N 2 B$ in type 2 diabetes [2-9]. Other successful studies have discovered loci related to a variety of disorders

\footnotetext{
* Correspondence: Kleinr@mskcc.org
'Program in Cancer Biology and Genetics, Memorial Sloan-Kettering Cancer

* Correspondence: kleinr@mskcc.org
'Program in Cancer Biology and Genetics, Memorial Sloan-Kettering Cancer Center, New York, NY 10065 USA
}

(c) 2011 Levenstien and Klein; licensee BioMed Central Ltd. This is an Open Access article distributed under the terms of the Creative lar disease [10-12].

Genome-wide association studies often utilize multiple stages in which promising signals are identified in an initial stage and followed up in subsequent stages. The process of follow up includes fine-mapping a signal using the same cohort and replicating the finding in additional cohorts. In both cases, SNPs need to be prioritized for selection in follow up studies since it is currently impractical to experimentally test every SNP at our disposal for association with disease. Some groups advocate prioritizing SNPs in genes and/or coding regions over other SNPs during selection [13]. By not only emphasizing nonsynonymous SNPs but also applying computational methods to detect nonsynonymous variants with the greatest potential to disrupt protein function, others take this strategy a step further [14-19]. Such prioritization begins to address the problem of an unwieldy number of SNPs to evaluate. 
Unfortunately, these gene-centric approaches fail to focus on functionally important SNPs outside of annotated genes. As will be justified below, a more comprehensive prioritization scheme, which considers SNPs outside of protein-coding regions, is warranted.

A compelling reason to consider a broader approach for SNP selection is that often genome-wide association studies have produced highly significant signals that fall in regions far from any annotated protein-coding gene. It is likely that these findings are true positives since in many cases independent research groups have replicated these results. According to the National Human Genome Research Institute's catalog of published GWA studies, to date greater than 460 associations have been found in intergenic regions or in regions where the gene is unknown. Presumably, many more associations are located in genomic regions devoid of annotated genes but are not reported in the catalog [1]. One puzzling example is the region at $8 \mathrm{q} 24$, which has been implicated in studies of prostate, breast, and colorectal cancer [3,20-25]. The closest gene, $M Y C$, is a viable candidate; however, it is more than $300 \mathrm{~kb}$ from the association signals. Another region implicated in multiple disorders is 9q21. Significant association signals in this region have been detected for myocardial infarction and type 2 diabetes and are $150 \mathrm{~kb}$ from the nearest genes [7-9,26-28].

Several researchers have applied approaches utilizing bioinformatics to investigate regions outside of coding regions for the existence of selective pressure. Chen et al. demonstrated significant negative selection in regions predicted as miRNA targets [29]. Similarly, others have shown negative selective pressure acting in predicted exon splicing enhancers, cis-elements, and introns [30-33]. Furthermore, negative selection has been identified in both conserved noncoding sequences and ultraconserved elements in the human genome although variations in these regions have not yet been linked to changes in phenotype [34-37]. A comprehensive study identifying genomic regions under negative selection utilizing a full array of the annotation currently available has not so far been undertaken. Such a study could provide the basis for a SNP prioritization scheme that extends beyond SNPs in coding regions.

Here we create a comprehensive scheme for prioritizing SNPs based on the likelihood that they are functional. Our approach involves identifying regions of the genome under negative selection. Since these regions appear intolerant of putatively deleterious alleles, they most likely harbor important functional features. We categorize the SNPs in the human genome based on the annotations available related to gene structure, crossspecies conservation, and regulatory elements. By examining the distribution of derived allele frequencies within each class, we assess the strength of evidence for negative selection in each class. We advocate employing this information to weight the SNPs based on their class affiliations. This approach targets SNPs in genomic regions that display evidence of function, regardless of whether protein-coding genes are found in the region.

\section{Results}

We classified SNPs in the Ensembl database into 44 classes based on the annotation available as detailed in the Methods section (Table 1, Additional File 1). An examination of these classes revealed that they are not mutually exclusive (Figure 1). Interestingly, the vast majority of SNPs in constrained elements, regions of the genome that show a high level of cross species conservation, did not appear in either coding regions (Figure 1B) or the regulatory features constructed by experimentally derived regulatory attributes (Figure 1C). These observations emphasize that constrained elements represent a distinct class worthy of separate consideration from coding and regulatory elements. Likewise, the majority of SNPs in coding regions and regulatory features did not reside in constrained elements (Figure $1 \mathrm{~B}$ and $1 \mathrm{C}$ ).

To identify regions of the genome under purifying selection, we compared the distribution of derived allele frequencies (DAF) in each class with that of the genome as a whole and, in a confirmatory analysis, with that of ancestral repeats, a well-accepted model of neutral selection [38-42]. For the comparisons, we utilized the allele frequencies from three populations comprehensively genotyped by Perlegen-African Americans (AFR), European Americans (EUR), and Han Chinese from the Los Angeles area (CHN) [43]. An excess of low derived

Table 1 Representative classes considered in this study

\begin{tabular}{lccc}
\hline class name & $\begin{array}{c}\text { number of } \\
\text { SNPs }\end{array}$ & $\begin{array}{c}\text { size of } \\
\text { region } \mathbf{( k b )}\end{array}$ & $\begin{array}{c}\text { SNP frequency } \\
\text { (SNPs/kb) }\end{array}$ \\
\hline coding & 132,562 & 34,215 & 3.87 \\
\hline promoter & 104,439 & 24,844 & 4.20 \\
\hline splice site & 41,932 & 11,621 & 3.61 \\
\hline $\begin{array}{l}\text { constrained } \\
\text { elements }\end{array}$ & 152,158 & 71,977 & 2.11 \\
\hline $\begin{array}{l}\text { regulatory features } \\
\text { core }\end{array}$ & 260,919 & 64,296 & 4.06 \\
\hline $\begin{array}{l}\text { regulatory features } \\
\text { extended }\end{array}$ & 558,932 & 136,134 & 4.11 \\
\hline cisRED & 9,491 & 2,815 & 3.37 \\
\hline miRanda & 3,975 & 884 & 4.50 \\
\hline $\begin{array}{l}\text { non-coding RNA } \\
\text { genes }\end{array}$ & 1,832 & 490 & 3.74 \\
\hline ancestral repeats & 44,243 & 13,011 & 3.40 \\
\hline genome & $11,307,522$ & $3,022,647$ & 3.74 \\
\hline SNP counts shown & & & \\
\hline
\end{tabular}

SNP counts shown are for all SNPs in Ensembl. 


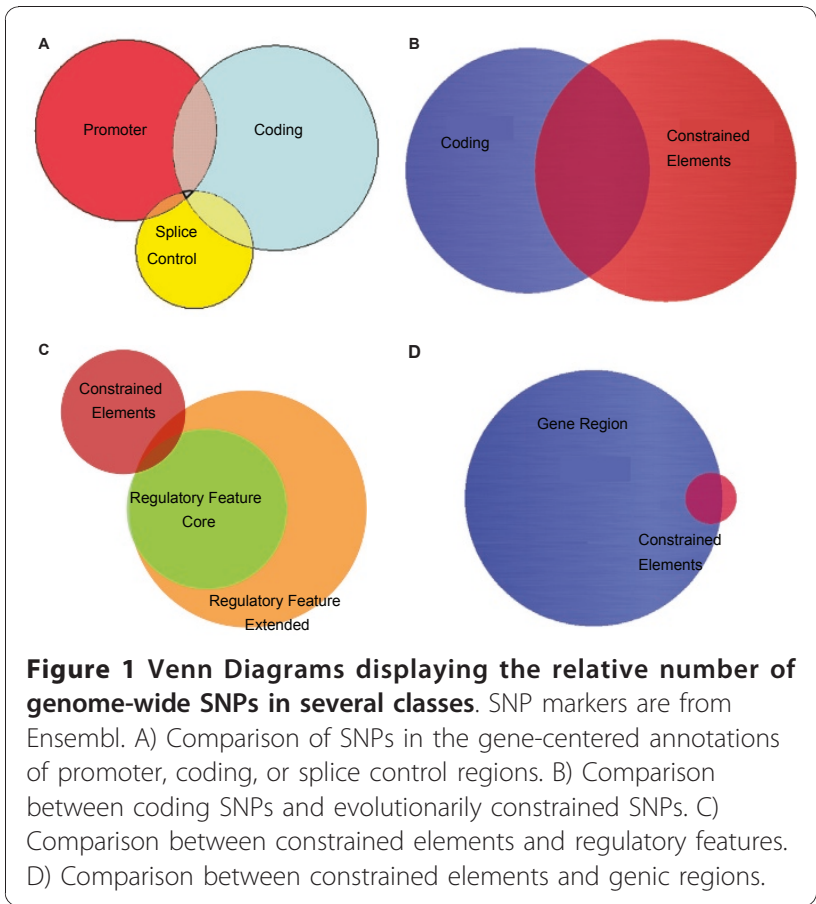

frequency SNPs in comparison to the genome (or ancestral repeats) implies a region is under negative selection.

We found evidence that suggests the existence of negative pressure for several classes. Seventeen classes were statistically significant (false discovery rate $<0.05$ ) in the AFR population (Table 2 and Additional File 1). Similar results were observed for the EUR and CHN populations (Additional File 1). We emphasize the AFR population because it possesses greater genetic diversity and many fewer monomorphic SNPs, thereby increasing our power to detect negative selection. As expected, protein-coding gene related classes such as nonsynonymous, coding, and splice site classes resided on the list of significant classes. Constrained element classes and regions where four specific histone methylations have been detected (H3K79me3, H3K36me3, H3K4me2, and H3K4me3) appeared statistically significant. Several other regulatory elements, including microRNA binding targets (miRanda elements), are also worth noting.

The above analysis rests on the assumption that the vast majority of the genome as a whole is under neutral selection and is therefore an appropriate benchmark for comparison. In order to validate this hypothesis, we utilized SNPs in ancestral repeats, a well-accepted model of neutral selection, in a second round of analyses for comparison [38-42]. The results from the comparisons with the ancestral repeats supported those from the comparisons with the genome as a whole. Six classes were statistically significant when compared to ancestral repeats for the Perlegen AFR population (Table 3 and
Table 2 Classes under negative selection compared to the genome

\begin{tabular}{clcc}
\hline rank & class name & $\boldsymbol{p}$-value & q-value \\
\hline 1 & coding & $<1 \times 10^{-8}$ & $1.5 \times 10^{-3}$ \\
\hline 2 & nonsynonymous & $<1 \times 10^{-8}$ & $3.1 \times 10^{-3}$ \\
\hline 3 & constrained elements & $<1 \times 10^{-8}$ & $4.7 \times 10^{-3}$ \\
\hline 4 & constrained elements minus coding & $<1 \times 10^{-8}$ & $6.3 \times 10^{-3}$ \\
\hline 5 & constrained elements minus genes & $<1 \times 10^{-8}$ & $7.8 \times 10^{-3}$ \\
\hline 6 & constrained elements 1 kb from genes & $<1 \times 10^{-8}$ & $9.4 \times 10^{-3}$ \\
\hline 7 & regulatory features extended & $<1 \times 10^{-8}$ & $1.1 \times 10^{-2}$ \\
\hline 8 & H3K36me3 & $<1 \times 10^{-8}$ & $1.3 \times 10^{-2}$ \\
\hline 9 & H3K79me3 & $<1 \times 10^{-8}$ & $1.4 \times 10^{-2}$ \\
\hline 10 & constrained elements 100 kb from genes & $1.0 \times 10^{-8}$ & $1.6 \times 10^{-2}$ \\
\hline 11 & splice site & $8.0 \times 10^{-4}$ & $1.7 \times 10^{-2}$ \\
\hline 12 & Dnasel & $4.5 \times 10^{-3}$ & $1.9 \times 10^{-2}$ \\
\hline 13 & H3K4me3 & $5.1 \times 10^{-3}$ & $2.0 \times 10^{-2}$ \\
\hline 14 & H3K4me2 & $8.6 \times 10^{-3}$ & $2.2 \times 10^{-2}$ \\
\hline 15 & Polll & $1.1 \times 10^{-2}$ & $2.3 \times 10^{-2}$ \\
\hline 16 & miRanda & $1.5 \times 10^{-2}$ & $2.5 \times 10^{-2}$ \\
\hline 17 & cisRED & $2.4 \times 10^{-2}$ & $2.7 \times 10^{-2}$ \\
\hline
\end{tabular}

Classes with a statistically significant excess of low derived alleles when compared to the genome as a whole are shown. In order to adjust for the multiplicity of testing, we apply an FDR correction with $\alpha=0.05$. Only resequenced Perlegen SNP markers are included in this analysis to minimize ascertainment bias. For our comparisons, we rely on allele frequencies present in the AFR Perlegen population.

Additional File 1). Again, the analyses using the EUR and $\mathrm{CHN}$ population allele frequencies showed similar results (Additional File 1). Similar to the comparison with the genome as a whole, the significant classes found in the comparison with ancestral repeats were the nonsynonymous and coding SNPs as well as all but one constrained elements classes. When comparing these results, it is important to recognize that the comparisons to the genome involved many more observations and, therefore, had a sizable power advantage to detect modest effects. Furthermore, we applied a less severe multiple test correction for the comparisons to the genome as a whole because we performed twelve fewer tests due to computational issues (see Methods).

Some borderline significant classes (unadjusted $p<$ 0.1 ) are also worth noting (Table 3 ). Interestingly, the H3K79me3 histone methylation class was extremely close to being significant. Albeit not as close as H3K79me3, the remaining constrained elements class, miRanda elements, and the H3K36me3 histone methylation class also seemed to provide strong evidence to support the findings from the initial comparison to the genome as a whole. Notably, several classes, including splice sites, Dnase I hypersensitivity sites, regulatory features extended (an Ensembl class summarizing the regulatory attributes), and $\mathrm{H} 3 \mathrm{~K} 4 \mathrm{me} 3$, which appeared 
Table 3 Classes under negative selection compared to ancestral repeats

\begin{tabular}{clcc}
\hline rank & class name & $p$-value & q-value \\
\hline 1 & nonsynonymous & $5.0 \times 10^{-8}$ & $1.1 \times 10^{-3}$ \\
\hline 2 & constrained elements & $3.6 \times 10^{-5}$ & $2.3 \times 10^{-3}$ \\
\hline 3 & constrained elements minus coding & $1.4 \times 10^{-4}$ & $3.4 \times 10^{-3}$ \\
\hline 4 & coding & $1.2 \times 10^{-3}$ & $4.5 \times 10^{-3}$ \\
\hline 5 & constrained elements minus genes & $3.0 \times 10^{-3}$ & $5.7 \times 10^{-3}$ \\
\hline 6 & constrained elements 1 kb from genes & $3.2 \times 10^{-3}$ & $6.8 \times 10^{-3}$ \\
\hline 7 & H3K79me3 & $8.0 \times 10^{-3}$ & $7.9 \times 10^{-3}$ \\
\hline 8 & constrained elements 100 kb from genes & $1.1 \times 10^{-2}$ & $9.0 \times 10^{-3}$ \\
\hline 9 & miRanda & $3.4 \times 10^{-2}$ & $1.0 \times 10^{-2}$ \\
\hline 10 & H3K36me3 & $4.0 \times 10^{-2}$ & $1.1 \times 10^{-2}$ \\
\hline 11 & Polll & $6.3 \times 10^{-2}$ & $1.3 \times 10^{-2}$ \\
\hline 12 & H3K4me2 & $7.1 \times 10^{-2}$ & $1.4 \times 10^{-2}$ \\
\hline 13 & cisRED & $1.0 \times 10^{-1}$ & $1.5 \times 10^{-2}$ \\
\hline
\end{tabular}

Classes with an excess of low derived alleles when compared to the ancestral repeats are shown. Bolded, italicized classes are statistically significant when we apply an FDR correction with $\alpha=0.05$. Only resequenced Perlegen SNP markers are included in this analysis to minimize ascertainment bias. For our comparisons, we rely on allele frequencies present in the AFR Perlegen population.

significant in the initial analysis did not appear significant or even borderline significant in the subsequent analysis involving the ancestral repeats. In particular, the regulatory features extended class may not appear significant in this subsequent analysis because this class is built from 24 distinct regulatory attributes - some displaying strong evidence of negative selection and other displaying little or no evidence of negative selection. While the regulatory features class as well as these other classes may still represent regions of the genome under negative selection and be functionally important, the confirmatory analysis utilizing ancestral repeats did not substantiate the initial findings.

As mentioned above, we observed evidence for negative selection among the SNPs in regulatory attributes, H3K79me3 and H3K36me3, as well as in constrained elements. We investigated the possibility that our observations were the result of an underlying hitchhiking effect or background selection. For the two regulatory attributes, we considered SNPs in random genomic regions comparably located to annotated genes (see
Methods). In this approach, we compared the $p$-values reported above, "real $p$-values", with the $p$-values generated using the random genomic regions, "generated p-values". For both the H3K79me3 and the H3K36me3 experiments, we found that the "real" $p$-values were considerably smaller than all 100 "generated" $p$-values in comparisons to both the genome and ancestral repeats (Table 4). In addition, we applied a FDR test correction to the "real" $p$-values to adjust for the multiplicity of testing involved in performing 44 tests. Again, the FDR adjusted $p$-values were smaller than all 100 "generated" $p$-values in comparisons to both the genome and ancestral repeats (Table 4). These results suggest that a hitchhiking effect or background selection cannot account for the observed negative selection in the regions containing these regulatory attributes.

For the constrained elements, we employ an alternative approach to ensure that our observation is not solely due to constrained element SNPs localized in coding regions. We considered subsets of the SNPs in the constrained element class that are located outside of genes, at least $1 \mathrm{~kb}$ from the closest gene, and at least $100 \mathrm{~kb}$ from the closest gene. In all three Perlegen populations, the derived allele frequencies of SNPs in constrained elements outside of genes were significantly lower than the derived allele frequencies in the genome as a whole and in ancestral repeats (Figure 2B; Table 5). We observed similar results when we only considered constrained elements at least $1 \mathrm{~kb}$ and constrained elements at least $100 \mathrm{~kb}$ from the closest gene relative to the genome as a whole and ancestral repeats (Figure 2C, Table 5). In summary, classes created by placing greater restrictions on the constrained elements class still display statistical significance.

As mentioned above, several classes derived from biological experiments have strong evidence of negative selection. For example, the derived allele frequencies in SNPs in regions known to have specific histone modifications had lower derived allele frequencies than SNPs in the genome as a whole or in ancestral repeats. Chief among these was H3K79me3 (Figure 2D; Table 5). This difference was statistically significant in all three populations when compared to the genome and in two of the populations when compared to ancestral repeats (Table 5).

Table 4 P-values and FDR adjusted $p$-values for the analyses involving regulatory attributes $\mathrm{H} 3 \mathrm{~K} 79 \mathrm{me} 3$ and H3K36me3

\begin{tabular}{lccc}
\hline & H3K79me3 & H3K36me3 & \\
\hline & ancestral repeats & genome & ancestral repeats \\
\hline$p$-value (rank) & $8.00 \times 10^{-3}(1)$ & $1.00 \times 10^{-8}(1)$ & $4.00 \times 10^{-2}(1)$ \\
\hline FDR adjusted $p$-value (rank) & $5.03 \times 10^{-2}(1)$ & $4.40 \times 10^{-7}(1)$ & $1.00 \times 10^{-8}(1)$ \\
\hline
\end{tabular}

Ranks for each "real" $p$-value are relative to a set of "generated" $p$-values produced by performing tests of evidence of selection on sets of SNPs in random genomic regions comparable, in size and proximity to annotated genes, to the regulatory feature under consideration. 


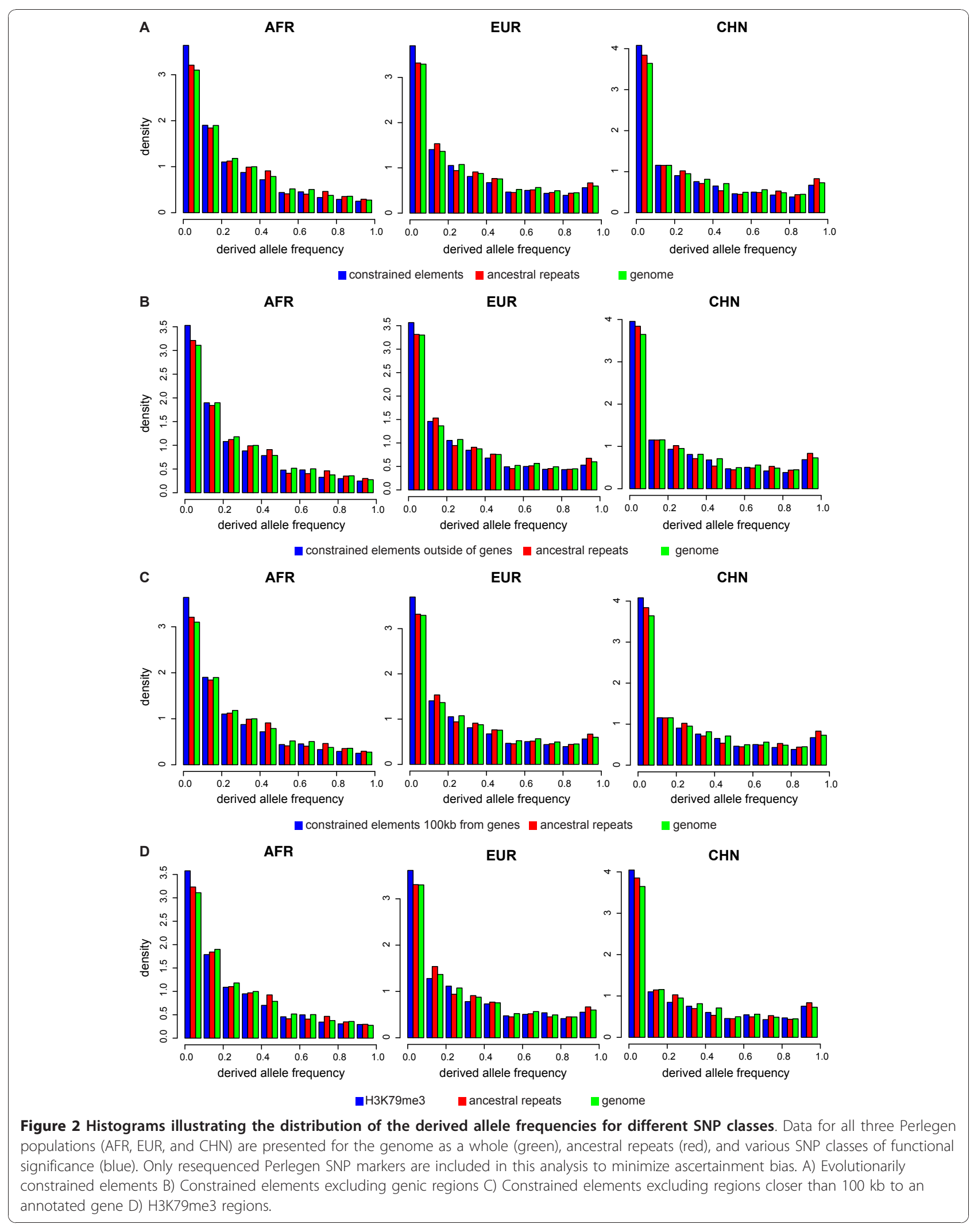


Table 5 Comparison of the distribution of derived allele frequencies (DAF) for SNPs within several classes

\begin{tabular}{|c|c|c|c|c|c|c|}
\hline \multirow[t]{2}{*}{ class } & \multirow[t]{2}{*}{ Perlegen population } & \multirow[t]{2}{*}{ class DAF median } & \multicolumn{2}{|c|}{ ancestral repeats } & \multicolumn{2}{|c|}{ genome } \\
\hline & & & DAF median & $p$-value & DAF median & $p$-value \\
\hline \multirow[t]{3}{*}{ constrained elements } & AFR & 0.174 & 0.196 & $3.6 \times 10^{-5}$ & 0.205 & $<1 \times 10^{-8}$ \\
\hline & EUR & 0.188 & 0.208 & $3.4 \times 10^{-4}$ & 0.229 & $<1 \times 10^{-8}$ \\
\hline & $\mathrm{CHN}$ & 0.174 & 0.208 & $8.7 \times 10^{-3}$ & 0.217 & $<1 \times 10^{-8}$ \\
\hline \multirow[t]{3}{*}{ constrained elements $1 \mathrm{~kb}$ from genes } & AFR & 0.174 & 0.196 & $3.2 \times 10^{-3}$ & 0.200 & $<1 \times 10^{-8}$ \\
\hline & EUR & 0.190 & 0.208 & $5.4 \times 10^{-3}$ & 0.229 & $<1 \times 10^{-8}$ \\
\hline & $\mathrm{CHN}$ & 0.188 & 0.208 & $6.0 \times 10^{-2}$ & 0.217 & $<1 \times 10^{-8}$ \\
\hline \multirow[t]{3}{*}{ constrained elements $100 \mathrm{~kb}$ from genes } & AFR & 0.174 & 0.196 & $1.1 \times 10^{-2}$ & 0.200 & $1.0 \times 10^{-8}$ \\
\hline & EUR & 0.205 & 0.208 & $9.6 \times 10^{-3}$ & 0.229 & $1.1 \times 10^{-6}$ \\
\hline & $\mathrm{CHN}$ & 0.188 & 0.208 & $8.3 \times 10^{-2}$ & 0.217 & $8.5 \times 10^{-7}$ \\
\hline \multirow[t]{3}{*}{ constrained elements outside of genes } & AFR & 0.174 & 0.196 & $3.0 \times 10^{-3}$ & 0.196 & $<1 \times 10^{-8}$ \\
\hline & EUR & 0.188 & 0.208 & $5.3 \times 10^{-3}$ & 0.229 & $<1 \times 10^{-8}$ \\
\hline & $\mathrm{CHN}$ & 0.188 & 0.208 & $6.1 \times 10^{-2}$ & 0.217 & $<1 \times 10^{-8}$ \\
\hline \multirow[t]{3}{*}{ H3K79me3 } & AFR & 0.174 & 0.196 & $8.0 \times 10^{-3}$ & 0.200 & $<1 \times 10^{-8}$ \\
\hline & EUR & 0.208 & 0.208 & $1.6 \times 10^{-2}$ & 0.229 & $1.7 \times 10^{-7}$ \\
\hline & $\mathrm{CHN}$ & 0.188 & 0.202 & $1.2 \times 10^{-1}$ & 0.217 & $5.0 \times 10^{-8}$ \\
\hline
\end{tabular}

Classes presented are 1) constrained elements, 2) constrained elements at least $1 \mathrm{~kb}$ from the closest gene, and 3) constrained elements at least $100 \mathrm{~kb}$ from the closest gene, 4) constrained elements outside of genes, and 5) H3K79me3 regulatory attributes in comparison with the genome as a whole and ancestral repeats. We perform a Mann-Whitney U-test to compare the DAF distribution for SNPs in constrained elements, constrained elements outside of genes, and H3K79me3 regulatory attributes with that of the genome and that of ancestral repeats. We list the resulting p-values as well as the median DAF for each class.

\section{Discussion}

Here we have shown that SNPs in several classes of genomic elements appear to be under negative selection. Most notably, we find that SNPs in constrained elements, even when $100 \mathrm{~kb}$ from the nearest gene, have a lower derived allele frequency than SNPs in presumably neutrally evolving regions. The constrained elements represent regions of the genome that are highly conserved among multiple species. Since it is thought that most SNPs arose subsequent to the last common ancestor of chimpanzees and humans, cross-species conservation in a region does not imply that the region is intolerant of new SNPs in humans. However, our work shows that an analysis of SNPs in human populations definitively demonstrates significant negative selection in these regions. Furthermore, since we find evidence for negative selection when the constrained elements are restricted to large distances from the closest annotated gene, we purport that constrained regions provide a meaningful class of putatively functionally important SNPs unto themselves without any contributions from known genes. These observations are in agreement with the findings of other groups that focused specifically on conserved regions rather than the broader approach we pursue [35-37].

Regulatory attributes represent another potentially useful group of classes for identifying functional SNPs. Our analysis finds two regulatory attributes, H3K79me3 and H3K36me3, with substantial support for negative selection. These attributes annotate regions of the genome where methylation of two specific lysines in histone H3 have been detected. The covalent addition of methyl groups to histones has been associated with both gene silencing and expression. The durability of the bond allows for epigenetic changes in gene expression that can be passed on to daughter cells. Whole genome scans of methylation sites in human cells revealed that many H3K36me3 methylations occur within transcribed regions of highly active genes [44,45]. Because this methylation peaks near the 3' end of active genes it has been hypothesized to play a role in RNA termination [46]. Whereas the H3K79me3 methylation is less well defined, several groups report a modest correlation with both highly expressed and silenced genes $[44,47]$. Regardless of their specific function, we believe that these regulatory attributes represent a refinement over a predicted promoter class since they are experimentally derived and displayed significant evidence of selection despite their small sample size.

In addition, other regulatory classes such as miRanda elements have some evidence of negative selection as well (Tables 2 and 3). miRanda is open-source software that predicts targets for microRNA-mediated translational repression in the 3 ' untranslated region of genes $[48,49]$. To date, the miRanda algorithm has identified 1,934,522 putative binding targets in 31,869 human gene isoforms for 677 currently known human microRNAs [50]. Although the set of rules for target prediction are not 
identical, our findings regarding microRNA binding targets concur with those of Chen et al. [29]. The miRanda algorithm incorporates current biological knowledge on target rules and relies on interspecies conservation. The miRanda class is distinctively different from the constrained elements as it does not apply the GERP scoring algorithm to the multiple sequence alignment across the same ten species. In addition, these regions represent an entity whose function is biologically distinct. However, it is not surprising that a class such as the miRanda class, which relies on interspecies conservation, shows evidence of negative selection. Overall, our analysis highlights specific regulatory elements that appear to have a greater potential to harbor functional SNPs.

Compared to the vast majority of classes we investigate, the individual regulatory elements contain relatively small sample sizes (Additional File 1). As with any statistical test, these small sample sizes negatively affect the power to detect a significant result. Even so, the miRanda elements and mRNA polymerase binding site (PolII), containing less than 300 and 1000 SNPs, respectively, show significant results when compared to the genome and borderline significant results when compared to ancestral repeats. Given that power is a function of sample size and magnitude of effect, in these cases a significant result suggests a substantial effect. Many of the other regulatory and non-protein coding RNA related classes are under powered due to their very small class membership (sample size $<50$ ) and may be shown to exhibit significant negative selection in future analysis with an enlarged sample. In contrast, other classes with substantial sample sizes such as CTCF, which contains almost 12,500 SNPs, are not significant in any analysis. Thus, the identification of significant classes does not simply follow linearly with increasing sample size.

Applying the information garnered from our analysis to address the problem of an unwieldy number of SNPs to evaluate in genome-wide association studies represents an additional challenge. Our analysis provides the foundation for a comprehensive SNP prioritization scheme to select markers meriting follow-up. Such follow-up includes replicating association results in independent cohorts as well as searching for causal variants in linkage disequilibrium with associated signals. Our data can also be used to weight specific association tests in the initial stage of a genome-wide association study. Several researchers have proposed approaches to weighting SNPs in genome-wide studies using biological information such as we present here. One strategy utilizes a Bayesian approach to weighting the hypotheses in an FDR framework [51-53]. Another approach relies on hierarchical modeling, which allows for multiple sources of prior information without prejudging their value
[54,55]. A third approach involves establishing groups of SNPs based on prior information and weighting them to optimize the average power of the study [56]. However, additional research is needed to determine the most powerful method. Regardless of the method, we strongly advocate harnessing the scientific insights we have found regarding genomic elements under negative selection to appropriately target regions most likely to harbor functional variants.

These data also have implications beyond the realm of genome-wide association studies. With the advent of next generation sequencing technologies, the affordability of large-scale genomic sequencing projects is rapidly increasing $[57,58]$. Ventures like the 1000 Genomes Project promise to provide whole-genome sequence for a large and diverse set of individuals [59]. Wholegenome sequencing has been used to identify both tumor-specific somatic mutations [60] and germline mutations in a Charcot-Marie-Tooth syndrome patient [61]. While these initial whole-genome resequencing studies focused on coding regions of annotated genes, a prioritization scheme could be employed as an efficient alternative to search for functional single nucleotide variants (SNVs) outside of coding regions. Since wholegenome sequencing will produce even more overwhelming amounts of data, the need to prioritize variants for follow-up will elevate in importance.

We expect in the future that many functional variants will be elucidated outside of the coding regions of known genes. In order to efficiently design studies to follow-up genome-wide association and whole genome sequencing efforts, we advocate a comprehensive prioritization scheme for variants based on evidence of negative selection. Our findings here illustrate the importance of considering genetic elements that lie outside of known protein-coding regions and highlight genomic elements which are most likely to contain variants that play a role in disease.

\section{Conclusions}

These data demonstrate that SNPs outside of coding regions, especially in evolutionarily conserved regions and in putative regulatory elements, appear to be under negative selection. Some such SNPs may have physiological consequences and be responsible for human phenotypic variation. These putative functional SNPs may be a good set of SNPs to examine first when trying to find the underlying mutation responsible for observed genetic associations.

\section{Methods}

We define classes based on the annotations available in the Ensembl database. Specifically, we access the annotations available in the Ensembl database through the 
Ensembl Perl application programming interface (API). This method allows us to extract all SNPs in specific regions relative to the various genome features, such as exons, transcripts, and genes, available through the database. We generate classes based on gene structure, conservation across species, and regulatory elements. For all of our analyses, we exclude SNPs which map to multiple positions in the human genome, map to the Y chromosome, or that do not appear in dbSNP. We retain SNPs mapping to alternative chromosome constructions or supercontigs if in addition they map to a single site on human chromosomes 1-22 or X.

\section{Gene Structure Based Classes}

We utilize the annotations from the Ensembl database (release 45) to locate the regions associated with each gene structure class relative to Ensembl known proteincoding genes. These classes include promoter, splice control, and coding regions. We define promoter regions as ranging from 1000 basepairs upstream of the transcription start site to 200 basepairs downstream of the transcription start site. We define splice control regions at intron/exon interfaces (intron upstream of interface/exon downstream of interface) as ranging from 50 basepairs upstream of the interface to 2 basepairs downstream of the interface and at exon/intron interfaces (exon upstream of interface/intron downstream of the interface) as ranging from 3 basepairs upstream of the interface to 6 basepairs downstream of the interface. This splice control region definition includes the polypyrimidine track and is based on the splice control consensus sequence in humans and related mammals. In addition, we utilize the Ensembl annotations available to locate non-coding RNA genes. Specifically, we generate classes for microRNA (miRNA), small nuclear RNA (snRNA), small nucleolar RNA (snoRNA), ribosomal RNA (rRNA), transfer RNA (tRNA), small cytoplasmic RNA (scRNA), and other miscellanous types of RNA (miscRNA).

\section{Conservation Based Class}

We utilize the results in Ensembl from the GERP scoring algorithm [62] to determine constrained elements which represent regions in the genome with a very high level of sequence conservation among the ten species (Mus musculus, Canis familiaris, Monodelphis domestica, Rattus norvegicus, Homo sapiens, Pan troglodytes, Gallus gallus, Bos taurus, Ornithorhynchus anatinus, Macaca mulatta) included in the multiple sequence alignment.

\section{Regulatory Elements Classes}

We utilize the annotations from the Ensembl database (release 47) to locate regulatory features, based on experimental evidence from genome-wide assays, and the underlying regulatory attributes used to construct them $[44,63]$. Ensembl contains annotations for 24 distinct regulatory attributes. In addition, we utilize the Ensembl annotations available to locate cisRED [64] and miRanda [50] regulatory elements.

\section{Distribution of Derived Allele Frequencies within Each Class}

To examine these classes for evidence of negative selection, we investigate the distribution of the derived allele frequencies (DAF) within each class. For each SNP, we find the orthologous base position in chimpanzee [29]. We define the "ancestral allele" as the chimpanzee allele equivalent in humans and the "derived allele" as the other human allele. In the event that the chimpanzee allele does not match either human allele, we compare the human alleles to the rhesus macaque reference sequence instead. For those SNPs where neither the chimpanzee nor the rhesus macaque allele matches one of the human alleles, we discard the SNP from the study. As a source for the allele frequencies, we use the publicly available genotype data from the Perlegen project populations-African Americans (AFR), European Americans (EUR), and Han Chinese from the Los Angeles area (CHN) [43]. We then compare the DAF distributions from the classes directly by employing a Mann-Whitney U-test as implemented by the software package R [65]. Specifically, we compare the DAF distributions for each class with that of the genome as a whole to detect purifying selection. As a confirmation, we repeat the comparison using the DAF for ancestral repeats, a widely accepted model of neutral selection, in place of the genome [38-42]. We define ancestral repeats using the method of Paten et al., which compares repeated sequence in five mammals (human, mouse, rat, dog, and cow), without consideration of ancestral repeat correspondence [66]. If the derived allele frequency spectrum in the class in question is lower than the derived allele frequency spectrum of the genome (or ancestral repeats), the DNA sequence in this class may be under negative selective pressure. While this approach is robust to mutation rate heterogeneity, it may be sensitive to ascertainment bias between functional regions of the genome. Ascertainment bias arises because in SNP discovery efforts often there is a bias towards SNPs in genes and common SNPs as these markers are located in well-studied regions of the genome or are more easily found. Although limiting our analysis to SNPs located in the HapMap ENCODE regions alleviates the ascertainment bias, sample sizes are compromised. As an alternative approach to minimize the ascertainment bias and maintain sample sizes, we limit the scope of the analysis to 
genome-wide SNPs that were resequenced by Perlegen [43]. Furthermore, for the DAF analyses, we consider only biallelic single base substitution SNPs and eliminate SNPs that overlap the two compared classes. Specifically, for comparisons to the genome, we eliminated the overlapping SNPs from the genome set only while for comparisons to ancestral repeats, we eliminated the overlapping SNPs from both classes in the comparison. After all comparisons, we determine statistical significance adjusting for the multiplicity of testing by applying the False Discovery Rate (FDR) correction with $\alpha=$ 0.05 [67]. We correct for 32 tests for comparisons to the genome as a whole while we correct for 44 tests for comparisons to ancestral repeats. The discrepancy in the number of tests occurs because of the necessity to use an alternative algorithm to compute accurate MannWhitney $\mathrm{U}$-test $p$-values when one of the two classes contains a small number of samples. While this approach works efficiently when the second class contains an intermediate number of samples (ancestral repeats class), it is computationally infeasible when the second class contains a very large number of samples (genome as a whole). Thus, we eliminate these comparisons from our analysis.

\section{Hitchhiking Effect}

We next explored the possibility that our observations are the result of an underlying hitchhiking effect or background selection. For each H3K79me3 regulatory attribute, we use the information concerning the size of the attribute and its distance to the closest annotated gene to define a region that is of identical size and distance from a randomly selected gene and, subsequently, mine this region for all SNPs. We pool the SNPs collected in this manner for all H3K79me3 regulatory attributes annotated in Ensembl and then perform two Mann Whitney tests on their DAFs against 1) the DAFs for the SNPs in the genome as a whole and 2) the DAFs for the SNPs in ancestral repeats. For these tests, we use the AFR Perlegen population to determine the frequency for each derived allele. We repeat this procedure for mining SNPs for 100 iterations and, consequently, generated 100 Mann-Whitney test $p$-values (for comparisons to the genome and 100 more $p$-values for the comparison to ancestral repeats). We compare these 100 "generated" $p$-values to the Mann-Whitney test $p$ value for the real H3K79me3 regulatory attributes and observe the number of "generated" $p$-values which are less than or equal to the "real" $p$-value. In addition, we perform this same experiment for the H3K36me3 regulatory attribute. The regulatory attributes, H3K79me3 and H3K36me3, showed the most evidence of negative selection (besides coding, nonsynonymous, and constrained element classes) and, therefore, we were most interested in ensuring these results were not due to an underlying hitchhiking effect.

\section{Additional material}

\begin{abstract}
Additional file 1: Evidence for negative selection among various annotation classes. Comparison of derived allele frequency for all classes against both ancestral repeats and the whole genome is shown for all three Perlegen populations. Yellow shaded classes are statistically significant when we apply an FDR correction with $\alpha=0.05$. Only resequenced Perlegen SNP markers are included in this analysis to minimize ascertainment bias. The file can be viewed with Microsoft Excel.
\end{abstract}

\section{Acknowledgements}

We thank P. Flicek and K. Beal for helpful discussions. This work was supported in part by the Society for the Memorial Sloan-Kettering Cancer Center and by MSKCC through NIH P30 CA008748.

\section{Authors' contributions}

MAL designed experiments, performed all analyses, and drafted the manuscript. RJK conceived of the study, designed experiments, interpreted results, and revised the manuscript. Both authors read and approved the final manuscript.

Received: 11 May 2010 Accepted: 19 January 2011

Published: 19 January 2011

\section{References}

1. A catalog of published genome-wide association studies. [http://www. genome.gov/gwastudies].

2. Altshuler D, Daly MJ, Lander ES: Genetic mapping in human disease. In Science. Volume 322. New York, NY; 2008:(5903):881-888.

3. Easton DF, Pooley KA, Dunning AM, Pharoah PD, Thompson D, Ballinger DG, Struewing JP, Morrison J, Field H, Luben R, et al: Genomewide association study identifies novel breast cancer susceptibility loci. Nature 2007, 447(7148):1087-1093.

4. Edwards AO, Ritter R, Abel KJ, Manning A, Panhuysen C, Farrer LA: Complement factor $\mathrm{H}$ polymorphism and age-related macular degeneration. In Science. Volume 308. New York, NY; 2005:(5720):421-424.

5. Haines $J$, Hauser MA, Schmidt S, Scott WK, Olson LM, Gallins P, Spencer KL, Kwan SY, Noureddine M, Gilbert JR, et al: Complement factor $\mathrm{H}$ variant increases the risk of age-related macular degeneration. In Science. Volume 308. New York, NY; 2005:(5720):419-421.

6. Klein RJ, Zeiss C, Chew EY, Tsai JY, Sackler RS, Haynes C, Henning AK, SanGiovanni JP, Mane SM, Mayne ST, et al: Complement factor H polymorphism in age-related macular degeneration. In Science. Volume 308. New York, NY; 2005:(5720):385-389.

7. Saxena R, Voight BF, Lyssenko V, Burtt NP, de Bakker PI, Chen H, Roix JJ, Kathiresan S, Hirschhorn JN, Daly MJ, et al: Genome-wide association analysis identifies loci for type 2 diabetes and triglyceride levels. In Science. Volume 316. New York, NY; 2007:(5829):1331-1336.

8. Scott L, Mohlke KL, Bonnycastle LL, Willer CJ, Li Y, Duren WL, Erdos MR, Stringham HM, Chines PS, Jackson AU, et al: A genome-wide association study of type 2 diabetes in Finns detects multiple susceptibility variants. In Science. Volume 316. New York, NY; 2007:(5829):1341-1345.

9. Zeggini E, Weedon MN, Lindgren CM, Frayling TM, Elliott KS, Lango $H$, Timpson NJ, Perry JR, Rayner NW, Freathy RM, et al: Replication of genome-wide association signals in UK samples reveals risk loci for type 2 diabetes. In Science. Volume 316. New York, NY; 2007:(5829):1336-1341

10. Easton DF, Eeles RA: Genome-wide association studies in cancer. Hum Mol Genet 2008, 17(R2):R109-115.

11. Lettre G, Rioux JD: Autoimmune diseases: insights from genome-wide association studies. Human molecular genetics 2008, 17(R2):R116-121.

12. Mohlke KL, Boehnke M, Abecasis GR: Metabolic and cardiovascular traits: an abundance of recently identified common genetic variants. Hum $\mathrm{Mol}$ Genet 2008, 17(R2):R102-108. 
13. Jorgenson E, Witte JS: A gene-centric approach to genome-wide association studies. Nat Rev Genet 2006, 7(11):885-891.

14. Karchin R, Diekhans M, Kelly L, Thomas DJ, Pieper U, Eswar N, Haussler D, Sali A: LS-SNP: large-scale annotation of coding non-synonymous SNPs based on multiple information sources. Bioinformatics 2005, 21(12):2814-2820.

15. Kashuk CS, Stone EA, Grice EA, Portnoy ME, Green ED, Sidow A, Chakravarti A, McCallion AS: Phenotype-genotype correlation in Hirschsprung disease is illuminated by comparative analysis of the RET protein sequence. Proc Natl Acad Sci USA 2005, 102(25):8949-8954.

16. Ng PC, Henikoff S: SIFT: Predicting amino acid changes that affect protein function. Nucleic Acids Res 2003, 31(13):3812-3814.

17. $\mathrm{Ng} \mathrm{PC}$, Henikoff S: Predicting the effects of amino acid substitutions on protein function. Annu Rev Genomics Hum Genet 2006, 7:61-80.

18. Sunyaev S, Ramensky V, Koch I, Lathe W, Kondrashov AS, Bork P: Prediction of deleterious human alleles. Hum Mol Genet 2001, 10(6):591-597.

19. Yue P, Melamud E, Moult J: SNPs3D: candidate gene and SNP selection for association studies. BMC Bioinformatics 2006, 7:166.

20. Amundadottir LT, Sulem P, Gudmundsson J, Helgason A, Baker A, Agnarsson BA, Sigurdsson A, Benediktsdottir KR, Cazier JB, Sainz J, et al: A common variant associated with prostate cancer in European and African populations. Nat Genet 2006, 38(6):652-658.

21. Freedman ML, Haiman CA, Patterson N, McDonald GJ, Tandon A, Waliszewska A, Penney K, Steen RG, Ardlie K, John EM, et al: Admixture mapping identifies $8 \mathrm{q} 24$ as a prostate cancer risk locus in AfricanAmerican men. Proc Natl Acad Sci USA 2006, 103(38):14068-14073.

22. Ghoussaini M, Song H, Koessler T, Al Olama AA, Kote-Jarai Z, Driver KE, Pooley KA, Ramus SJ, Kjaer SK, Hogdall E, et al: Multiple loci with different cancer specificities within the 8q24 gene desert. J Natl Cancer Inst 2008, 100(13):962-966

23. Haiman CA, Le Marchand L, Yamamato J, Stram DO, Sheng X, Kolonel LN, Wu AH, Reich D, Henderson BE: A common genetic risk factor for colorectal and prostate cancer. Nat Genet 2007, 39(8):954-956

24. Tomlinson IP, Webb E, Carvajal-Carmona L, Broderick P, Howarth $K$, Pittman AM, Spain S, Lubbe S, Walther A, Sullivan K, et al: A genome-wide association study identifies colorectal cancer susceptibility loci on chromosomes 10p14 and 8q23.3. Nat Genet 2008, 40(5):623-630.

25. Zanke BW, Greenwood CM, Rangrej J, Kustra R, Tenesa A, Farrington SM Prendergast J, Olschwang S, Chiang T, Crowdy E, et al: Genome-wide association scan identifies a colorectal cancer susceptibility locus on chromosome 8q24. Nat Genet 2007, 39(8):989-994.

26. Helgadottir A, Thorleifsson G, Manolescu A, Gretarsdottir S, Blondal T, Jonasdottir A, Jonasdottir A, Sigurdsson A, Baker A, Palsson A, et al: A common variant on chromosome $9 \mathrm{p} 21$ affects the risk of myocardial infarction. In Science. Volume 316. New York, NY; 2007:(5830):1491-1493.

27. McPherson R, Pertsemlidis A, Kavaslar N, Stewart A, Roberts R, Cox DR, Hinds DA, Pennacchio LA, Tybjaerg-Hansen A, Folsom AR, et al: A common allele on chromosome 9 associated with coronary heart disease. Science 2007, 316(5830):1488-1491

28. Samani NJ, Erdmann J, Hall AS, Hengstenberg C, Mangino M, Mayer B, Dixon RJ, Meitinger T, Braund P, Wichmann HE, et al: Genomewide association analysis of coronary artery disease. N Engl J Med 2007, 357(5):443-453.

29. Chen K, Rajewsky N: Natural selection on human microRNA binding sites inferred from SNP data. Nat Genet 2006, 38(12):1452-1456.

30. Fairbrother WG, Holste D, Burge CB, Sharp PA: Single nucleotide polymorphism-based validation of exonic splicing enhancers. PLOS Biol 2004, 2(9):E268.

31. Sethupathy P, Giang H, Plotkin JB, Hannenhalli S: Genome-wide analysis of natural selection on human cis-elements. PloS one 2008, 3(9):e3137.

32. Davidson S, Starkey A, MacKenzie A: Evidence of uneven selective pressure on different subsets of the conserved human genome; implications for the significance of intronic and intergenic DNA. BMC genomics 2009, 10:614

33. Lomelin $\mathrm{D}$, Jorgenson $\mathrm{E}$, Risch $\mathrm{N}$ : Human genetic variation recognizes functional elements in noncoding sequence. Genome research 2010, 20(3):311-319.

34. Chen $C T$, Wang JC, Cohen BA: The strength of selection on ultraconserved elements in the human genome. Am J Hum Genet 2007, 80(4):692-704.
35. Drake JA, Bird C, Nemesh J, Thomas DJ, Newton-Cheh C, Reymond A, Excoffier L, Attar H, Antonarakis SE, Dermitzakis ET, et al: Conserved noncoding sequences are selectively constrained and not mutation cold spots. Nat Genet 2006, 38(2):223-227.

36. Katzman S, Kern AD, Bejerano G, Fewell G, Fulton L, Wilson RK, Salama SR, Haussler D: Human genome ultraconserved elements are ultraselected. In Science. Volume 317. New York, NY; 2007:(5840):915.

37. Goode DL, Cooper GM, Schmutz J, Dickson M, Gonzales E, Tsai M, Karra K, Davydov E, Batzoglou S, Myers RM, et al: Evolutionary constraint facilitates interpretation of genetic variation in resequenced human genomes. Genome research 2010, 20(3):301-310.

38. Ellegren $\mathrm{H}$, Smith $\mathrm{NG}$, Webster MT: Mutation rate variation in the mammalian genome. Curr Opin Genet Dev 2003, 13(6):562-568.

39. Gibbs RA, Weinstock GM, Metzker ML, Muzny DM, Sodergren EJ, Scherer S Scott G, Steffen D, Worley KC, Burch PE, et al: Genome sequence of the Brown Norway rat yields insights into mammalian evolution. Nature 2004, 428(6982):493-521.

40. Hardison RC, Roskin KM, Yang S, Diekhans M, Kent WJ, Weber R, Elnitski L, $\mathrm{Li}$ J, O'Connor M, Kolbe D, et al: Covariation in frequencies of substitution, deletion, transposition, and recombination during eutherian evolution. Genome Res 2003, 13(1):13-26.

41. Waterston RH, Lindblad-Toh K, Birney E, Rogers J, Abril JF, Agarwal P, Agarwala R, Ainscough R, Alexandersson M, An P, et al: Initial sequencing and comparative analysis of the mouse genome. Nature 2002, 420(6915):520-562

42. Yang S, Smit AF, Schwartz S, Chiaromonte F, Roskin KM, Haussler D, Miller W, Hardison RC: Patterns of insertions and their covariation with substitutions in the rat, mouse, and human genomes. Genome Res 2004 14(4):517-527.

43. Hinds DA, Stuve LL, Nilsen GB, Halperin E, Eskin E, Ballinger DG, Frazer KA, Cox DR: Whole-genome patterns of common DNA variation in three human populations. In Science. Volume 307. New York, NY; 2005:(5712):1072-1079.

44. Barski A, Cuddapah S, Cui K, Roh TY, Schones DE, Wang Z, Wei G, Chepelev I, Zhao K: High-resolution profiling of histone methylations in the human genome. Cell 2007, 129(4):823-837.

45. Boyle AP, Davis S, Shulha HP, Meltzer P, Margulies EH, Weng Z, Furey TS, Crawford GE: High-resolution mapping and characterization of open chromatin across the genome. Cell 2008, 132(2):311-322

46. Bannister AJ, Schneider R, Myers FA, Thorne AW, Crane-Robinson C, Kouzarides T: Spatial distribution of di- and tri-methyl lysine 36 of histone $\mathrm{H3}$ at active genes. J Biol Chem 2005, 280(18):17732-17736.

47. Wang Z, Zang C, Rosenfeld JA, Schones DE, Barski A, Cuddapah S, Cui K, Roh TY, Peng W, Zhang MQ, et al: Combinatorial patterns of histone acetylations and methylations in the human genome. Nat Genet 2008 40(7):897-903

48. Enright AJ, John B, Gaul U, Tuschl T, Sander C, Marks DS: MicroRNA targets in Drosophila. Genome Biol 2003, 5(1):R1.

49. John B, Enright AJ, Aravin A, Tuschl T, Sander C, Marks DS: Human MicroRNA targets. PLoS Biol 2004, 2(11):e363.

50. Betel D, Wilson M, Gabow A, Marks DS, Sander C: The microRNA.org resource: targets and expression. Nucleic Acids Res 2008, 36(Database issue):D149-153

51. Benjamini $Y$, Hochberg Y: Multiple hypothesis testing with weights. Scand J Statist 1997, 24:407-418.

52. Genovese $C R$, Roeder $\mathrm{K}$, Wasserman L: False discovery control with $\mathrm{p}$ value weighting. Biometrika 2006, 93(3):509-524.

53. Roeder K, Bacanu SA, Wasserman L, Devlin B: Using linkage genome scans to improve power of association in genome scans. Am J Hum Genet 2006, 78(2):243-252.

54. Chen GK, Witte JS: Enriching the analysis of genomewide association studies with hierarchical modeling. American journal of human genetics 2007, 81(2):397-404.

55. Lewinger JP, Conti DV, Baurley JW, Triche TJ, Thomas DC: Hierarchical Bayes prioritization of marker associations from a genome-wide association scan for further investigation. Genet Epidemiol 2007, 31(8):871-882

56. Roeder K, Devlin B, Wasserman L: Improving power in genome-wide association studies: weights tip the scale. Genetic epidemiology 2007 31(7):741-747. 
57. Kahvejian A, Quackenbush J, Thompson JF: What would you do if you could sequence everything? Nat Biotechnol 2008, 26(10):1125-1133.

58. Shendure J, Ji H: Next-generation DNA sequencing. Nat Biotechnol 2008, 26(10):1135-1145.

59. 1000 Genomes. [http://www.1000genomes.org/]

60. Ley TJ, Mardis ER, Ding L, Fulton B, McLellan MD, Chen K, Dooling D, Dunford-Shore BH, McGrath S, Hickenbotham M, et al: DNA sequencing of a cytogenetically normal acute myeloid leukaemia genome. Nature 2008, 456(7218):66-72

61. Lupski JR, Reid JG, Gonzaga-Jauregui C, Rio Deiros D, Chen DC, Nazareth L, Bainbridge $M$, Dinh $H$, Jing $C$, Wheeler DA, et al: Whole-genome sequencing in a patient with Charcot-Marie-Tooth neuropathy. N Engl J Med 2010, 362(13):1181-1191.

62. Cooper GM, Stone EA, Asimenos G, Green ED, Batzoglou S, Sidow A: Distribution and intensity of constraint in mammalian genomic sequence. Genome Res 2005, 15(7):901-913.

63. Kim TH, Abdullaev ZK, Smith AD, Ching KA, Loukinov DI, Green RD, Zhang MQ, Lobanenkov W, Ren B: Analysis of the vertebrate insulator protein CTCF-binding sites in the human genome. Cell 2007, 128(6):1231-1245.

64. Robertson G, Bilenky M, Lin K, He A, Yuen W, Dagpinar M, Varhol R, Teague K, Griffith OL, Zhang X, et al: cisRED: a database system for genome-scale computational discovery of regulatory elements. Nucleic Acids Res 2006, 34(Database issue):D68-73.

65. Akashi $\mathrm{H}$ : Inferring the fitness effects of DNA mutations from polymorphism and divergence data: statistical power to detect directional selection under stationarity and free recombination. Genetics 1999, 151(1):221-238

66. Paten B, Herrero J, Beal K, Fitzgerald S, Birney E: Enredo and Pecan: genome-wide mammalian consistency-based multiple alignment with paralogs. Genome research 2008, 18(11):1814-1828.

67. Benjamini $Y$, Hochberg $Y$ : Controlling the false discovery rate: a practical and powerful approach to multiple testing. J Roy Statist Soc B 1995 57(1):289-300

doi:10.1186/1471-2105-12-26

Cite this article as: Levenstien and Klein: Predicting functionally

important SNP classes based on negative selection. BMC Bioinformatics $201112: 26$

\section{Submit your next manuscript to BioMed Central} and take full advantage of:

- Convenient online submission

- Thorough peer review

- No space constraints or color figure charges

- Immediate publication on acceptance

- Inclusion in PubMed, CAS, Scopus and Google Scholar

- Research which is freely available for redistribution

Submit your manuscript at www.biomedcentral.com/submit 